\title{
Functional assessment of a left coronary-pulmonary artery fistula by coronary flow reserve
}

\author{
Viktor Sasi, Attila Nemes, Tamás Forster, Imre Ungi \\ Division of Invasive Cardiology, Department of Cardiology, Medical Faculty, Albert Szent-Györgyi Clinical Center, University of Szeged, \\ Szeged, Hungary
}

Postep Kardiol Inter 2014; 10, 2 (36): 141-143

DOI: 10.5114/pwki.2014.43526

\begin{abstract}
We report a 71-year-old man who presented with atypical chest pain. Coronary angiography did not reveal left main or proximal left anterior descending coronary artery stenosis, but a fistulous communication with a stronger tube-like fistula was present originating from the proximal left anterior descending coronary artery and emptying into the main pulmonary artery. Fractional flow reserve and coronary flow reserve measurements were performed to gain more data on the potential functional aspects of this fistula. With the present case, the importance of functional evaluation of these fistulas is demonstrated.
\end{abstract}

Key words: coronary, fistula, flow, fractional, pulmonary artery, reserve.

\section{Case report}

A 71-year-old man presented with atypical chest pain. Physical examination revealed a continuous murmur (1/6) over the apex. Electrocardiogram showed sinus rhythm with no noteworthy abnormalities. Transthoracic echocardiography indicated good ejection fraction with no wall motion abnormalities, hypertrophic left ventricle, slightly enlarged left atrium and grade I mitral regurgitation. A turbulent flow could be demonstrated in the distal part of the left main coronary artery (LM) and in the proximal left anterior descending coronary artery (LAD) by Doppler echo. Coronary angiography was performed but neither LM nor LAD stenosis could be confirmed; however, fistulous communications with a stronger tube-like fistula was present originating from the proximal LAD emptying into the main pulmonary artery (PA) (Figures 1 and 2) [1, 2]. The pulmonary-to-systemic flow ratio (Qp/Qs) was 1.1/1. Data were obtained from oxygen saturation samples from systemic arterial blood, left atrial/pulmonary venous blood, pulmonary artery, and caval vein/right heart samples. The single photon emission computed tomography (SPECT) thallium stress test demonstrated discrete persistent perfusion defects on the anterior and posterior walls with no transient component. In agreement with the coronary angiography finding, computer tomography angiography revealed a fistula of $1 \mathrm{~mm}$ diameter.

In order to assess the functional importance of these fistulous communications, fractional flow reserve (FFR) and coronary flow reserve (CFR) measurements were performed in the donor artery with a Pressure Wire System (St. Jude Medical, Minneapolis, MN, USA). Measurements were performed with administration of $50 \mathrm{U} / \mathrm{kg}$ heparin. To minimize vasoaction and measurement variability, $200 \mu \mathrm{g}$ of intracoronary (i.c.) nitroglycerin was also injected 3 min before the sensor guide-wire was advanced. The amount of i.c. adenosine was administered going up from $200 \mu \mathrm{g}$ to $240 \mu \mathrm{g}$ for each FFR measurement. For CFR assessments, $20 \mathrm{mg}$ of papaverine was administered i.c. The LAD-FFR was found to be 0.86, LAD-CFR was 1.56. The CXFFR and CX-CFR were 0.97 and 2.33, respectively. In view of these results and anatomic features, neither covered stent deployment nor coil embolization was performed.

\section{Discussion}

The majority of patients with coronary artery fistulas have no symptoms, but dyspnea, congestive heart fail-

Corresponding author:

Attila Nemes MD, PhD, FESC, $2^{\text {nd }}$ Department of Medicine and Cardiology, Medical Faculty, Albert Szent-Györgyi Clinical Center, University of Szeged, H-6720 Szeged, Korányi fasor 6, Hungary P.O. Box 427, phone: +36-62-545220, fax: +36-62-544568,

e-mail: nemes.attila@med.u-szeged.hu

Received: 18.03.2014, accepted: 8.05.2014. 

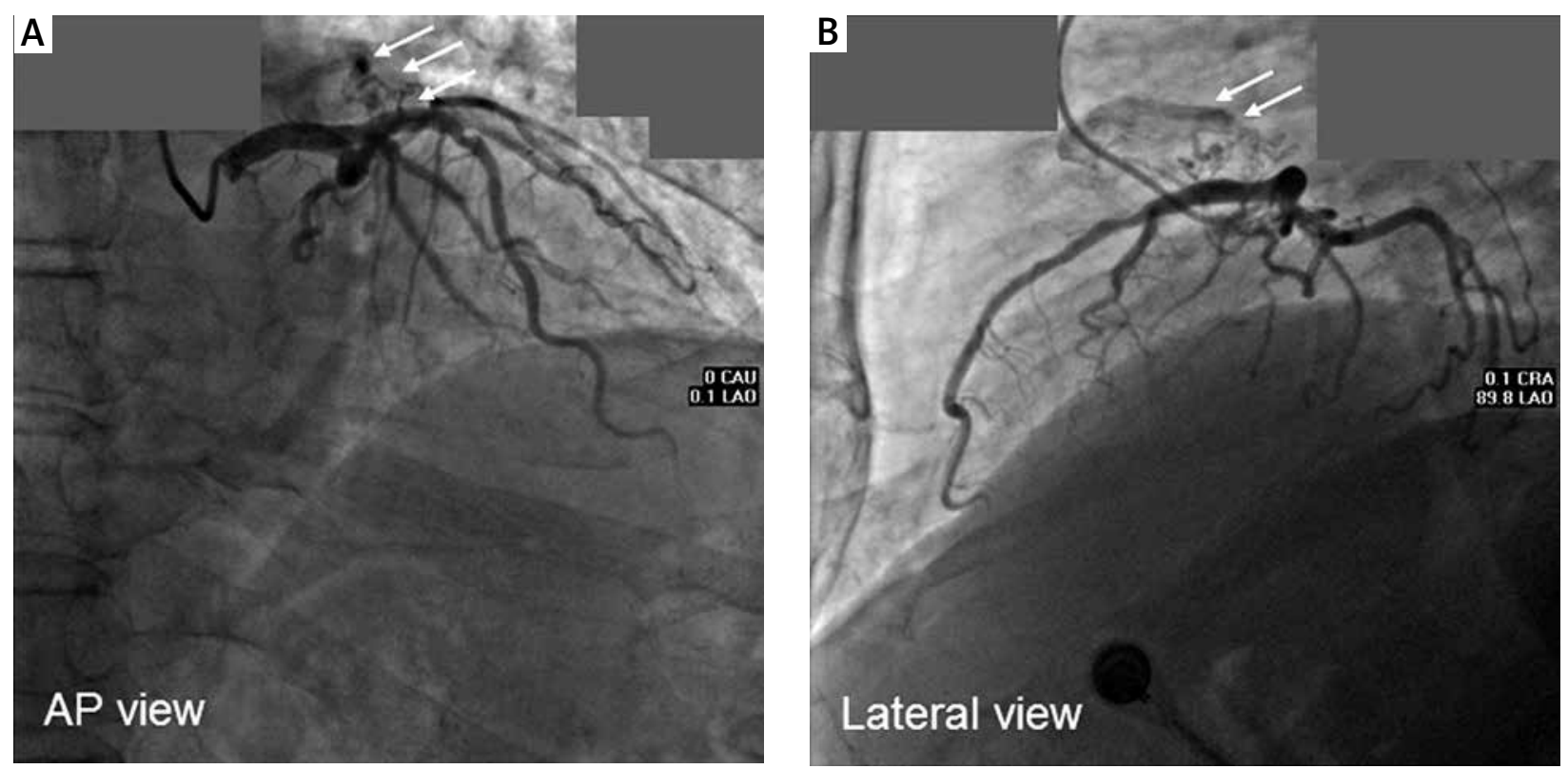

Figure 1. Anteroposterior projection of the left coronary system showing contrast opacification towards the main pulmonary artery through numerous micro-fistulas and one stronger tube-like fistula originating from the proximal part of the left anterior descending artery (LAD) (A, see white arrows). Lateral view of the left coronary system showing contrast media transported towards the main pulmonary artery through micro-fistulas and one stronger channel originating from the proximal LAD (B, see white arrows).
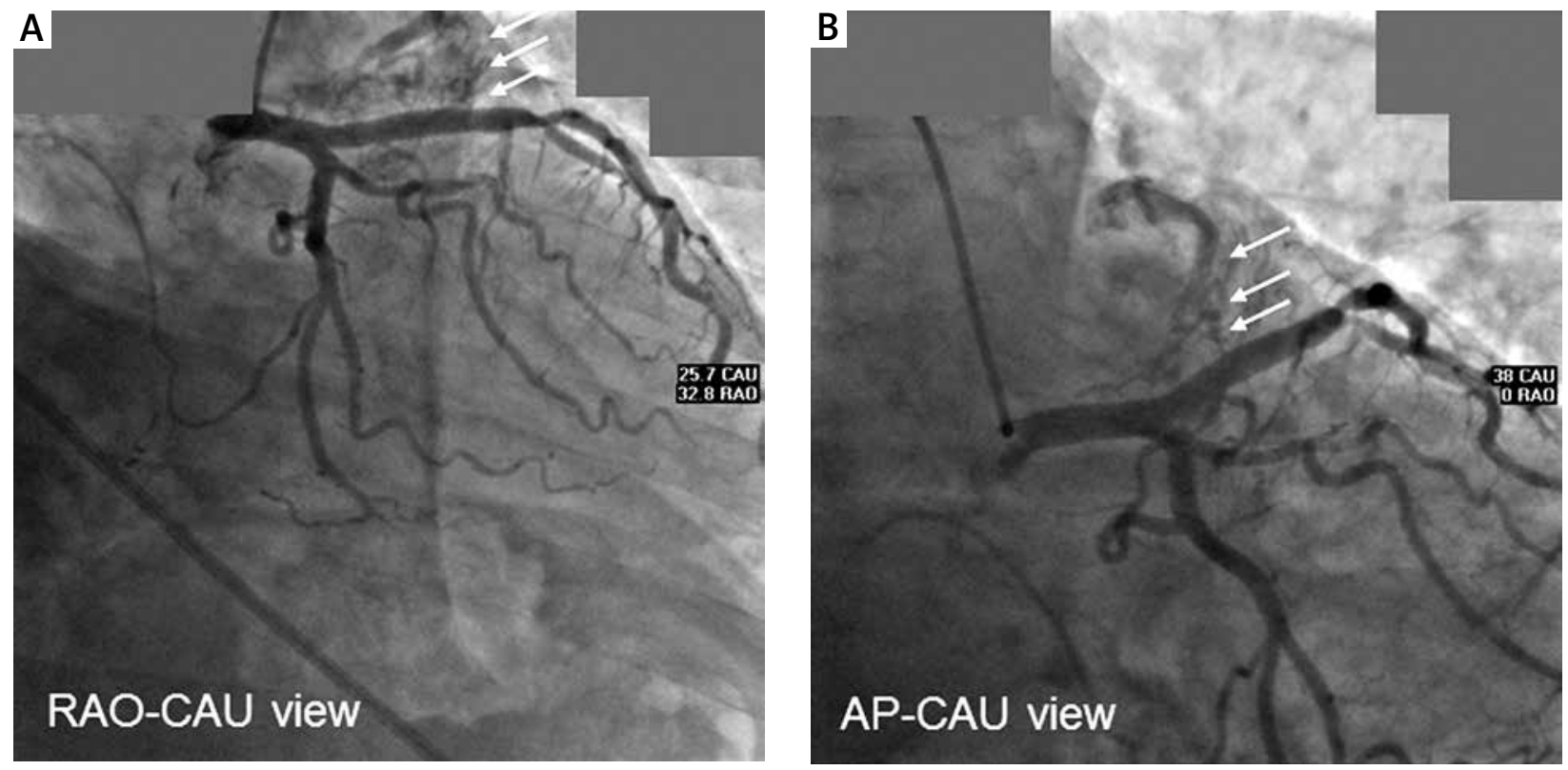

Figure 2. Right anterior oblique-caudal view of the left coronary system with fistulas apparent towards the main pulmonary artery originating from the proximal part of the left anterior descending artery (LAD) (A, see white arrows). Anteropostero-caudal view of the left coronary system showing numerous micro-fistulas and one stronger tube-like fistula from the proximal LAD with contrast material transported towards the main pulmonary artery (B, see white arrows)

ure, angina pectoris, endocarditis, arrhythmia, or myocardial infarction may be present $[1,2]$. The non-invasive diagnosis of such fistulas is often difficult; therefore coronary angiography is currently the standard method of choice for the diagnosis of this phenomenon [1-5]. In cases of fistulous communications from the coronary tree the flow in the donor vessel can be compromised, especially in the presence of a proximal fistulous channel. In 
normal coronary arteries, there is no apparent pressure loss along the length of the coronary artery. In case of a stenosis, the distal coronary pressure decreases due to the resistance of the stenosis; however, perfusion pressure can be possibly lost via fistulas as well. It has been postulated that FFR may be feasible for functional severity assessment of coronary fistulas $[4,5]$. In this case a low FFR indicates a "pseudo-stenosis" of the anatomically normal vessels.

The shunt blood flow under resting conditions can provide useful extra information only if the shunt ratio is above 1.5 [1, 2]. At maximum hyperemia, coronary steal can be manifested especially if the donor vessel supplies the territory of a dominant vessel. Harle et al. demonstrated that temporary occlusion of the fistula results in normalized FFR in the donor vessel; thus it was assumed that FFR measurement is a feasible means of diagnosing the functional severity in patients with a possible coronary steal [5].

It has been proved by simultaneous FFR and CFR determinations in patients with chronic total occlusion that a coronary steal through the collateral vessels influenced the functional severity of the disease in the donor vessel [6]. In our patient, however, the case was completely different because the donor artery supplied an extracardiac compartment, i.e. the pulmonary circulation, in which the blood flow supposedly did not increase under the adenosine effect. The slightly decreased LAD-FFR might be explained by the turbulence at the origin of the fistula, which may have resulted in some impediment of the flow. An angiographically undetectable non-significant proximal LAD lesion may also have explained this difference.

Coronary resistance is controlled by arterioles and small arteries. The absolute CFR is the ratio of the maximum hyperemic to the basal mean coronary flow velocity in the target vessel. The CFR expresses the capacity of a two-component system of a coronary artery and the supplied vascular bed to achieve maximum blood flow in response to a given hyperemic stimulation [3]. In the present case, the LAD-CFR was remarkably below the cutoff value of 2 , while the CX-CFR was normal. This phenomenon can be explained by the fact that a significant fraction of the blood is diverted from the distal LAD into the pulmonary circulation by the collaterals, and this flow was not affected by the i.c. papaverine injection. Hereby the effective increase of the myocardial flow in the distal LAD stayed behind that of the CX artery. In other words, the relative decrease of CFR in the LAD reflected the relative flow in the fistula compared to the fraction effectively supplying the myocardial territory of the vessel. An essential limitation of this explanation is that the flow of the proximal and distal LAD and furthermore that of the pulmonary circulation was not directly measured. However, this deficiency resulted from the thermodilu- tion method of CFR measurement that we applied. There are numerous factors that contribute to the fact that CFR cannot be utilized in clinical practice directly. The CFR values even in normal coronary arteries can be diverse, but if the value exceeds $15 \%$, it is considered to be significant as in the case presented. In the case presented the CXCFR value was normal; therefore we could rule out other humoral, neurological factors and endothelial dysfunction for the abnormal LAD-CFR result.

It cannot yet be stated whether an invasive or surgical strategy or a conservative approach would result in a better outcome for these patients. The anatomic defect may be corrected surgically [2] or percutaneously with coils or covered stents $[7,8]$. Though the decrease of CFR in the LAD denoted a remarkable steal to the pulmonary circulation, in consideration of the missing evidence of myocardial ischemia, and the potential complications of surgical ligation or intervention we proceeded with medical therapy $[2,7]$.

\section{Conclusions}

It may be stated that a thermodilution CFR measurement can allow a functional evaluation of coronary-pulmonary fistulas.

\section{Acknowledgments}

Dr. Attila Nemes holds a János Bolyai Research Fellowship (Budapest, Hungary).

\section{References}

1. Said SA, van der Werf T. Dutch survey of coronary artery fistulas in adults: congenital solitary fistulas. Int J Cardiol 2006; 106: 323-32.

2. Gowda RM, Vasavada BC, Khan IA. Coronary artery fistulas: clinical and therapeutic considerations. Int J Cardiol 2006; 107: 7-10.

3. Lim MJ, Kern MJ. Coronary pathophysiology in the cardiac catheterization laboratory. Curr Probl Cardiol 2006; 31: 493-550.

4. Sasi V, Ungi I, Forster T, Nemes A. Multiple coronary fistulas originating from all major coronary arteries. Acta Cardiol 2010; 65: 357-60.

5. Harle T, Kronberg K, Elsasser A. Coronary artery fistula with myocardial infarction due to steal syndrome. Clin Res Cardiol 2012; 101: 313-5.

6. Werner GS, Fritzenwanger M, Prochnau D, et al. Determinants of coronary steal in chronic total coronary occlusions donor artery, collateral, and microvascular resistance. J Am Coll Cardiol 2006; 48: 51-8.

7. Armsby LR, Keane JF, Sherwood MC, et al. Management of coronary artery fistulae. Patient selection and results of transcatheter closure. J Am Coll Cardiol 2002; 39: 1026-32.

8. Katona A, Ungi I, Forster T, et al. A single coronary artery with a right ventricular fistula: unique therapy with covered-stent implantation. Herz (in press). 